\title{
ANÁLISE DE GRUPOS DE PESQUISA EM ATIVIDADE FISICA E ENVELHECIMENTO NO BRASIL
}

\author{
Joilson Meneguci ${ }^{1}$ \\ Rafaela Gomes Santos² \\ Douglas Assis Teles Santos ${ }^{3}$ \\ Renata Damião ${ }^{4}$ \\ Jair Sindra Virtuoso Júnior \\ Edmar Lacerda Mendes ${ }^{6}$
}

\footnotetext{
1 Graduado em Educação Física. Mestre em Educação Física. Professor Substituto da Universidade Federal do Triângulo Mineiro (UFTM), vinculado ao Departamento de Ciências do Esporte. E-mail: joilsonmeneguci@yahoo.com.br.

2 Graduada em Educação Física. Mestre em Educação Física. Professora da Faculdade de Ciências Humanas e Sociais - AGES, vinculada ao Departamento de Educação Física. E-mail: rafaellagomes_rgds@hotmail.com.

3 Graduado em Educação Física. Mestre em Educação Física. Professor Auxiliar da Universidade do Estado da Bahia (UNEB), Campus X - Teixeira de Freitas, vinculado ao Departamento de Educação Física. E-mail: funnyeduca@hotmail.com.

4 Graduada em Nutrição. Doutora em Medicina (Endocrinologia Clínica). Professora Adjunta da Universidade Federal do Triângulo Mineiro (UFTM), vinculada ao Departamento de Nutrição. E-mail: renatadamiao@nutricao.uftm.edu.br.

5 Graduado em Educação Física. Doutor em Ciências da Saúde. Professor Adjunto da Universidade Federal do Triângulo Mineiro (UFTM), vinculado ao Departamento de Ciências do Esporte. E-mail: jair@ef.uftm.edu.br.

6 Graduado em Educação Física. Doutor em Biologia Celular e Estrutural. Professor Adjunto da Universidade Federal do Triângulo Mineiro (UFTM), vinculado ao Departamento de Ciências do Esporte E-mail: edmar@ef.uftm.edu.br.
} 
resumo

Atividade física regular tem sido recomendada pelos estudiosos da área do envelhecimento devido aos seus benefícios à saúde. O objetivo desse estudo foi analisar e caracterizar os Grupos de Pesquisa em Atividade Física e Envelhecimento no Brasil, de acordo com a distribuição geográfica. Realizou-se busca sistemática no Diretório de Grupos de Pesquisa do Conselho Nacional de Desenvolvimento Científico e Tecnológico sobre a temática: Atividade Física e Envelhecimento. Os Grupos de Pesquisa em Atividade Física e Envelhecimento certificados estão distribuídos entre as regiões Sudeste $(n=8)$, Sul $(n=6)$ e Nordeste $(n=4)$. Artigos publicados em periódicos representam 69,7\% ( $n=639)$ da produção científica da região Sudeste, 65,0\% (n=39) da região Sul e 64,9\% ( $n=126)$ da região Nordeste. Observou-se um gradativo aumento no número de Grupos de Pesquisa em Atividade Física e Envelhecimento no Brasil, porém estes grupos ainda não estão distribuídos por todo país.

palavras-chave

Atividade física. Envelhecimento. Produção Científica. Ciências da Saúde.

\section{Introdução}

O envelhecimento da população está relacionado a um grande desafio para a área da Saúde Pública, sendo necessária atenção às doenças crônicas não transmissíveis e políticas públicas voltadas à promoção de saúde (LEBRÃO, 2007; KALACHE, 2008). O Instituto Brasileiro de Geografia e Estatística, em 2010, registrou 20.590 .599 pessoas com 60 anos ou mais, o que representa 10,8\% do total da população (IBGE, 2010a). De acordo com projeções populacionais baseadas no Censo de 2010, a quantidade de pessoas com idade igual ou superior a 60 anos continuará aumentando, podendo chegar a $73.551 .010 \mathrm{em}$ 2060, representando 33,7\% da população (IBGE, 2013).

A relação entre atividade física regular e longevidade ganha destaque nos diversos campos das Ciências da Saúde devido aos benefícios reportados pela literatura científica ao longo dos anos (GILLUM; OBISESAN, 2010; ALMEIDA et al., 2014). O elevado e crescente contingente de idosos (SCULLY, 2012) e a redução da mortalidade associada ao estilo de vida fisicamente ativo (BROWN et al., 2012) têm estimulado pesquisas sobre os benefícios da atividade física no envelhecimento. 
Uma das formas de identificar quais são os grupos de pesquisa no Brasil que têm o objetivo de estudar a relação da atividade física com o envelhecimento é através do Diretório de Grupos de Pesquisa do Conselho Nacional de Desenvolvimento Científico e Tecnológico (CNPq). O Diretório de Grupos de Pesquisa cadastra grupos de pesquisadores, estudantes e pessoal de apoio técnico organizado em torno de linhas de pesquisa segundo regra hierárquica constituída na experiência e na competência técnico-científica (BRASIL, 2013a). Este, portanto, tem importante papel na preservação da memória da atividade científico-tecnológica em nosso país e constitui ferramenta para interação/ troca de informações. Suas bases de dados (Base Corrente e Base Censitária) trazem informações diretamente disponíveis sobre os grupos; por exemplo, localização e produções científicas nos últimos anos. Seu caráter censitário convida ao aprofundamento do conhecimento por meio das inúmeras possibilidades de estudos de tipo survey. Assim, o Diretório de Grupos de Pesquisa é um eficiente instrumento para o planejamento e a gestão das atividades de ciência e tecnologia (BRASIL, 2013a).

De forma a determinar a produção do conhecimento e a disseminação das informações geradas no campo da atividade física e envelhecimento, faz-se necessário identificar o surgimento dos Grupos de Pesquisa em Atividade Física e Envelhecimento (GPAFEs), a distribuição no país e sua produção científica. Em nosso conhecimento, informações sobre a atuação de grupos de pesquisas na área da saúde e em específico sobre atividade física e envelhecimento, nas distintas regiões brasileiras, são escassas (MENDES; ANDAKI; ANDAKI JÚNIOR, 2011; BALBÉ et al., 2012). Assim, o objetivo do presente estudo foi analisar e caracterizar GPAFEs no Brasil, de acordo com a distribuição geográfica.

\section{Metodologia}

Estudo sistemático e descritivo, realizado na base de dados do Diretório de Grupos de Pesquisa do CNPq sobre a temática Atividade Física e Envelhecimento, durante o mês de novembro de 2012.

Foi utilizada como estratégia de busca a frase exata "Atividade Física e Envelhecimento". Em seguida, foi identificado o ano de formação, distribuição geográfica, recursos humanos (estudantes, pesquisadores e técnicos), quantidade de líderes e linhas de pesquisa dos GPAFEs. A titulação dos líderes e sua respectiva produção científica foram consultadas no Currículo Lattes. Foram consideradas as produções científicas a partir do ano de formação do GPAFE. 
A certificação do GPAFE pelos dirigentes institucionais de pesquisa foi adotada como critério de inclusão para análises. A certificação dos grupos de pesquisa é de responsabilidade dos dirigentes das atividades de pesquisa da instituição a que o líder do grupo está vinculado (BRASIL, 2013a).

As informações recolhidas dos GPAFEs e no currículo Lattes dos pesquisadores foram tabulados no programa Excel versão 2010. Para apresentação dos resultados foram utilizados procedimentos da estatística descritiva, por meio de frequência absoluta e relativa.

\section{Resultados}

Foram identificados 25 grupos de pesquisas a partir do descritor exato "Atividade Física e Envelhecimento", todos da grande área de conhecimento denominada de Ciências da Saúde. Do total dos GPAFEs, sete não estavam certificados pelos dirigentes institucionais de pesquisa. Na Figura 1 são apresentadas as áreas de conhecimento relacionadas aos GPAEFs certificados.

Figura 1 - Áreas de conhecimento dos Grupos de Pesquisa em Atividade Física e Envelhecimento, 2012.

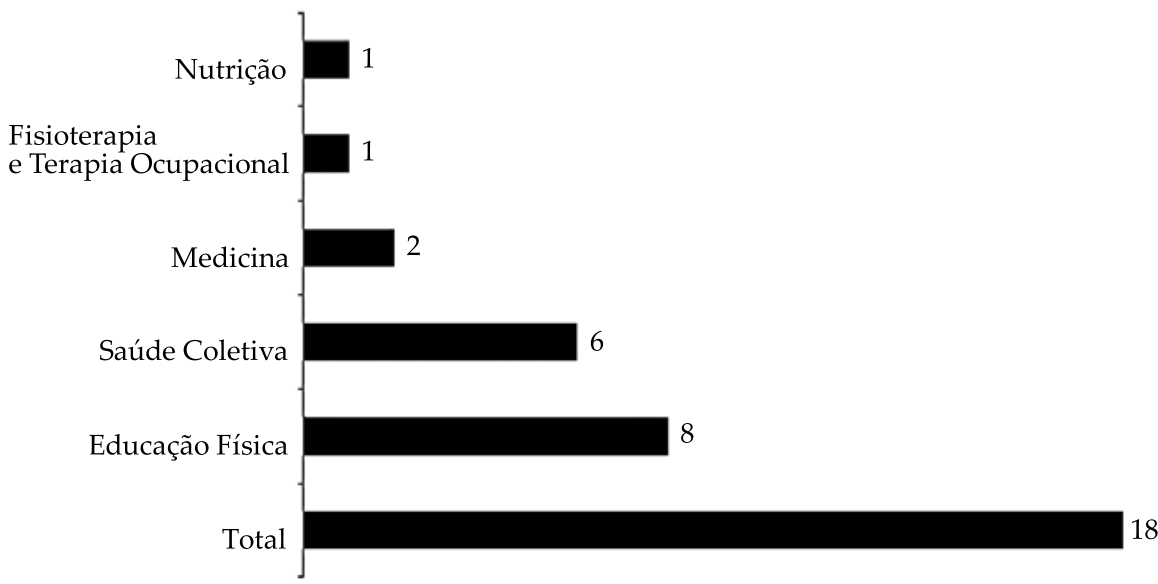

Em relação aos grupos não certificados, três são da área de conhecimento da Medicina, dois da Saúde Coletiva e dois da Educação Física de acordo com a CAPES. 
O número total dos GPAFEs certificados está distribuído em oito estados brasileiros, sendo eles: Bahia, Minas Gerais, Paraná, Pernambuco, Rio de Janeiro, Rio Grande do Sul, Santa Catarina e São Paulo, abrangendo três regiões do Brasil (Figura 2). Os dezoito GPAFEs encontravam-se inseridos em dezesseis diferentes Instituições de Ensino Superior, sendo dez instituições públicas e seis privadas.

Figura 2 - Distribuição geográfica dos Grupos de Pesquisa em Atividade Física e Envelhecimento no Brasil, 2012. Os valores foram expressos em frequência absoluta por estado e região e frequência relativa por região.

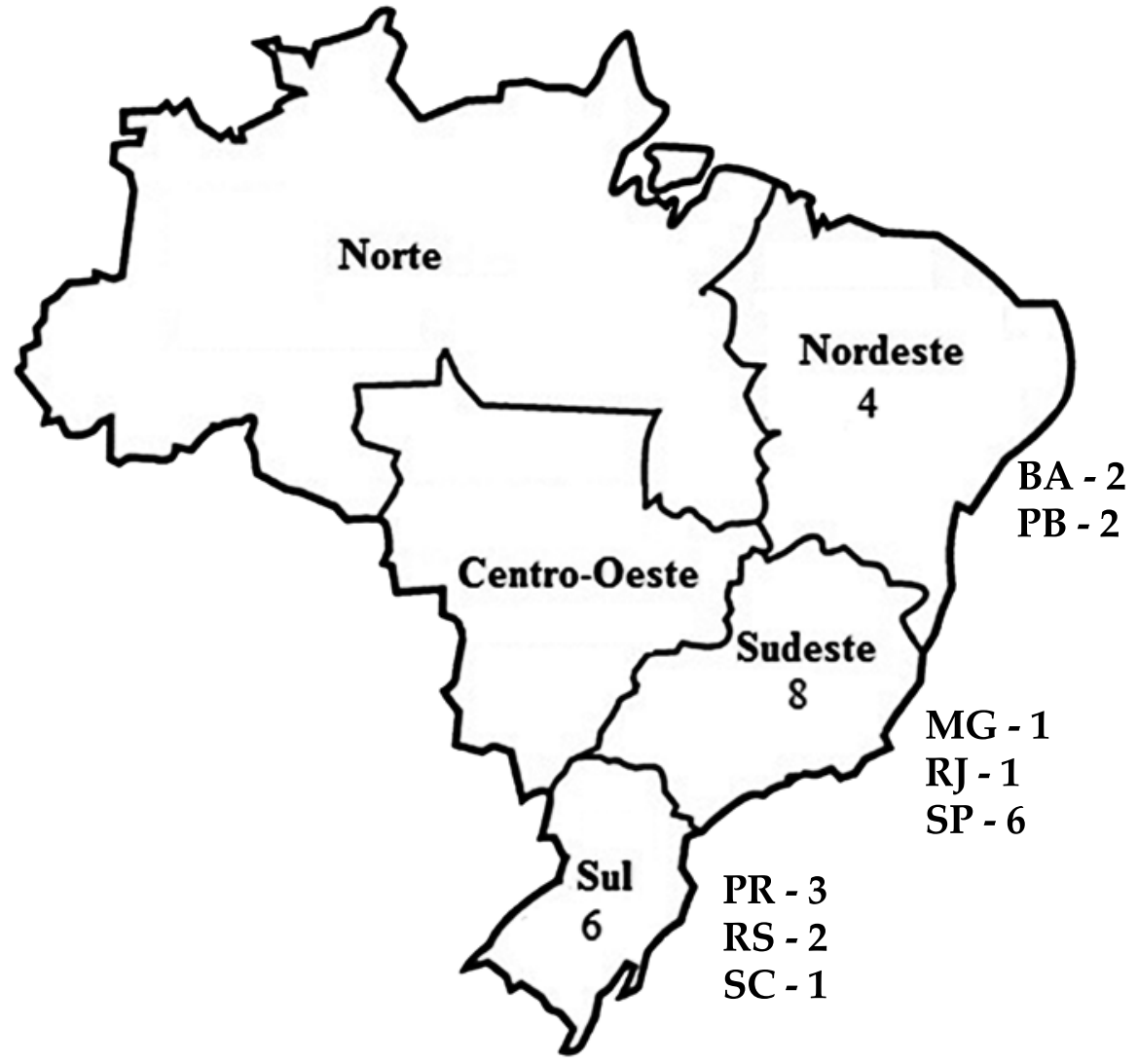

A Figura 3 apresenta o surgimento dos GPAFEs. O GPAFE mais antigo foi formado em 1983, sendo que cinco grupos foram criados entre 1983 a 2001, seis grupos, entre 2002 a 2007, e sete grupos foram criados entre 2008 a 2012. 


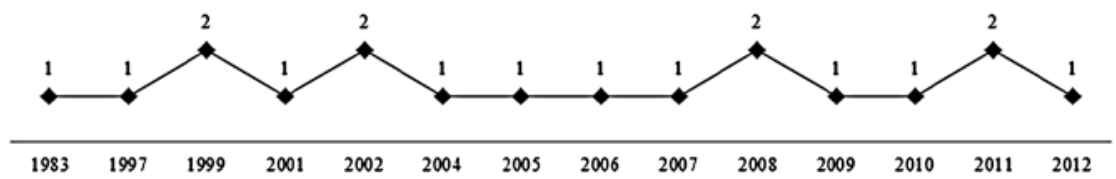

Os 18 GPAFEs identificados desenvolvem 72 linhas de pesquisa; destes grupos, nove apresentam de 1 a 3 linhas de pesquisa, oito apresentam entre 4 a 6, e um grupo apresenta 11 linhas de pesquisa. Das 72 linhas de pesquisa, 34 estão na região Sudeste, 24 estão na região Sul e 14 estão na região Nordeste.

Foram incluídos nas análises 31 líderes, pois 13 GPAFEs encontrados são liderados por dois pesquisadores. Apenas três líderes apresentaram titulação de mestrado, enquanto que 28 são doutores. Quando comparada com as demais regiões, a titulação dos líderes da região Sudeste se destaca, sendo que 14 deles são doutores (Figura 4).

Figura 4 - Distribuição da titulação dos líderes dos Grupos de Pesquisa em Atividade Física e Envelhecimento de acordo com as regiões do Brasil, 2012.

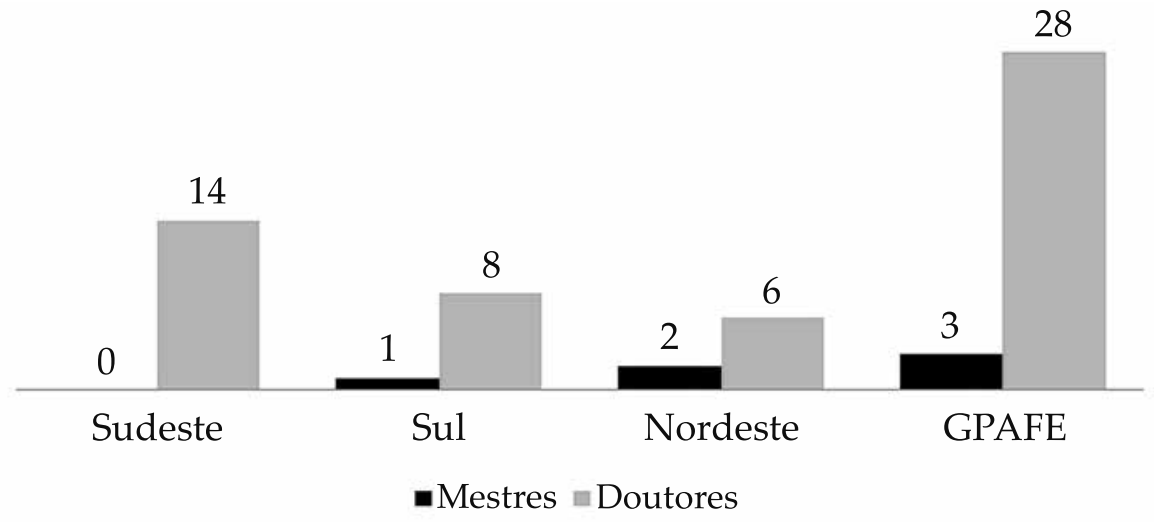

A partir do Currículo Lattes dos pesquisadores líderes, foi verificada a produção científica dos GPAFEs; tal produção foi identificada por meio de artigos publicados em periódicos indexados, livros publicados ou organizados, capítulos de livros, trabalhos completos publicados em anais de congressos e artigos aceitos para publicação, a partir do ano de criação do grupo de pesquisa. 
A Tabela 1 apresenta a distribuição das publicações de acordo com as regiões do Brasil e a Figura 5 demonstra, de acordo com as áreas de conhecimento, as publicações dos líderes dos GPAFEs.

Tabela 1 - Distribuição da produção científica dos líderes de Grupos de Pesquisa em Atividade Física e Envelhecimento por regiões do Brasil, 2012.

\begin{tabular}{l|c|c|c|c}
\hline \multicolumn{1}{c|}{ Tipo de produção científica } & $\begin{array}{c}\text { Sudeste } \\
(\mathbf{n}=\mathbf{8})\end{array}$ & $\begin{array}{c}\text { Sul } \\
(\mathbf{n}=\mathbf{6})\end{array}$ & $\begin{array}{c}\text { Nordeste } \\
(\mathbf{n}=\mathbf{4})\end{array}$ & $\begin{array}{c}\text { GPAFE } \\
(\mathbf{n}=\mathbf{1 8})\end{array}$ \\
\hline Artigos completos em periódicos & 639 & 39 & 126 & 804 \\
\hline Livros publicados/organizados ou edições & 31 & 2 & 4 & 37 \\
\hline Capítulos de livros publicados & 143 & 4 & 18 & 165 \\
\hline $\begin{array}{l}\text { Trabalhos completos publicados em anais } \\
\text { de congressos }\end{array}$ & 78 & 11 & 22 & 111 \\
\hline Artigos aceitos para publicação & 26 & 4 & 24 & 54 \\
\hline
\end{tabular}

De acordo com a Tabela 1, os artigos publicados em periódicos correspondem a $68,7 \%(\mathrm{n}=804)$ de toda produção científica dos GPAFEs, representando $69,7 \%(n=639)$ da produção científica da região Sudeste, $65,0 \%(n=39)$ da região Sul e $64,9 \%(n=126)$ da região Nordeste.

Figura 5 - Publicação dos líderes dos Grupos de Pesquisa em Atividade Física e Envelhecimento de acordo com as áreas de conhecimento, 2012.

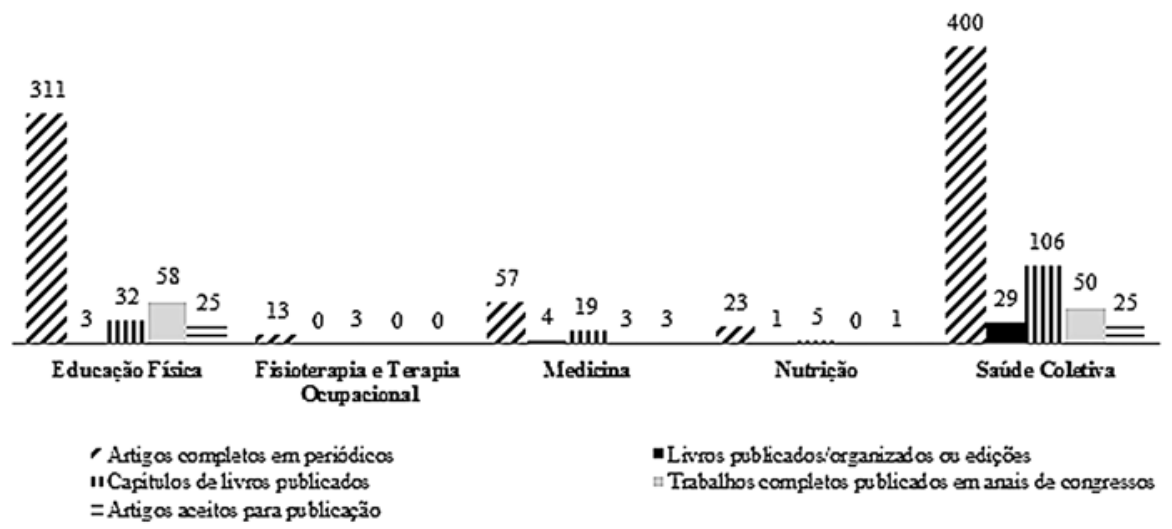


Em relação à distribuição das publicações de acordo com as áreas de conhecimento, a Saúde Coletiva $(52,1 \%$; $n=610)$, seguida pela Educação Física $(36,6 \% ; n=429)$ foram as áreas com maior concentração de produção dos GPAFEs (Figura 5).

Os líderes, pesquisadores, discentes de graduação e pós-graduação e o pessoal de apoio técnico constituem os recursos humanos encontrados nos GPAFEs. A Tabela 2 relaciona os integrantes dos GPAFEs envolvidos em cada região do Brasil.

Tabela 2 - Distribuição dos membros dos Grupos de Pesquisa em Atividade Física e Envelhecimento por regiões do Brasil, 2012.

\begin{tabular}{l|c|c|c|c}
\hline \multicolumn{1}{c|}{ Região } & Pesquisadores & Estudantes & Técnicos & $\begin{array}{c}\text { Total dos } \\
\text { Integrantes }\end{array}$ \\
\hline Sudeste & 97 & 118 & 8 & 223 \\
\hline Sul & 47 & 53 & 7 & 107 \\
\hline Nordeste & 42 & 46 & 3 & 91 \\
\hline GPAFE & 186 & 217 & 18 & 421 \\
\hline
\end{tabular}

A região Sudeste concentra o maior número de integrantes $(53,0 \% ; \mathrm{n}=223)$ dos GPAFEs no Brasil, seguidos pelas regiões Sul $(25,4 \%, \mathrm{n}=107)$ e Nordeste $(21,6 \% ; n=91)$. Os GPAFEs são constituídos por estudantes de graduação e pós-graduação $(51,5 \% ; n=217)$, seguido pelos docentes pesquisadores $(44,2 \%$; $\mathrm{n}=186)$ e técnicos $(4,3 \% ; \mathrm{n}=18)$.

\section{Discussão}

Evidências epidemiológicas confirmam que a prática regular de atividade física está relacionada a benefícios para saúde durante o processo de envelhecimento. Dessa forma, o interesse pela temática Atividade Física e Envelhecimento ganha evidência no cenário científico nacional (MATSUDO; MATSUDO; BARROS NETO, 2001; MATSUDO, 2006). Entretanto, as regiões Norte e Centro-Oeste do país apresentam defasagem em GPAFEs cadastrados e, consequentemente, baixa produção científica na área.

É válido ressaltar que o processo de transição demográfica não se deu de forma homogênea em todo território nacional; por exemplo, os dados da Pesquisa Nacional por Amostra de Domicílios - PNAD, realizada em 2009, revelam que as regiões Norte e Centro-Oeste apresentam menor população de 
idosos em relação às demais (IBGE, 2010b). Certamente, este indicador reflete significativamente em muitas análises que envolvem a população idosa por regiões brasileiras.

Diferentemente da não abrangência de todas as regiões pelos GPAFEs, os grupos de pesquisa que discutem a temática Atividade Física e Saúde estão presentes em todas as regiões do Brasil (MENDES; ANDAKI; ANDAKI JÚNIOR, 2011), mas aqueles com linhas de pesquisa sobre envelhecimento humano também não estavam inseridos na região Norte (PRADO; SAYD, 2004).

Os GPAFEs estão concentrados na região Sudeste, seguido do Sul e Nordeste. Possivelmente, este fato pode estar relacionado ao número elevado dos cursos de pós-graduação (Mestrado e Doutorado) na grande área da Ciência da Saúde e área da Educação Física, especialmente no estado de São Paulo, onde foi evidenciado o maior número de GPAFEs (BRASIL, 2013b).

De acordo com os resultados apresentados, os GPAFEs estão inseridos em áreas de conhecimento distintas da Educação Física e esse fato pode ser explicado pelo impacto da transição demográfica, principalmente pelo crescimento acelerado da população idosa representar um dos maiores desafios para a área da Saúde Pública (VERAS, 2009).

A região Sudeste possui o maior número de líderes dos GPAFEs com titulação de doutores, seguido do Sul e Nordeste. Apenas as regiões Sul e Nordeste apresentaram líderes com titulação de mestrado. Este fato pode ser explicado, em parte, pelo maior incentivo e avanço dos cursos de pós-graduação na região Sudeste (BRASIL, 2013b).

O grupo Envelhecimento e Saúde UnATI-UERJ (Universidade Estadual do Rio de Janeiro) é o mais antigo da temática Atividade Física e Envelhecimento, fundado em 1983 e inserido na área da Ciência Coletiva. Os primeiros GPAFEs inseridos na subárea Educação Física (abrange atualmente o maior número de GPAFEs) surgiram na década de 1990. Porém, estes grupos de pesquisa não são aqueles que mais têm produzido artigos científicos, de acordo com a análise dos currículos Lattes dos líderes. É válido ressaltar que não foi verificado se a produção dos líderes condiz com a temática em discussão e o fato de um grupo estar inserido nesta temática não significa que a produção de conhecimento sobre esse tema tenha iniciado.

De acordo com revisão de literatura realizada por Matsudo, Matsudo e Barros Neto (2001), a prática regular de atividade física está relacionada a benefícios durante o processo de envelhecimento. Na preocupação com o aumento da população idosa e em função da busca de um envelhecimento bem-sucedido, programas de promoção de atividade física têm surgido a cada 
momento (MATSUDO, 2006; ANDRÉA et al., 2010). Paralelamente ao aumento de programas destinados à promoção da saúde, pesquisas relacionadas à temática Atividade Física e Envelhecimento na Pós-graduação Stricto Sensu em Educação Física também tem aumentado, principalmente em nível de mestrado (BALBÉ et al., 2012).

Balbé et al. (2012) analisaram as produções científicas sobre atividade física e envelhecimento nas dissertações e teses dos programas de pós-graduação em Educação Física, no Brasil, até o ano de 2008, e identificaram que a discussão da temática tem se dado principalmente por dissertações de mestrado a partir de 1996.

No presente estudo, a análise da distribuição da produção científica dos líderes dos GPAFEs, demonstrou que a publicação de artigos em periódicos científicos foi o principal meio para veiculação do conhecimento produzido, observado para todas as subáreas do conhecimento de abrangência dos GPAFEs. Além disso, a produção de artigos em periódicos científicos pelos GPAFEs está concentrada em maior número na região Sudeste, seguida do Nordeste e Sul. Esse tipo de produção facilita a veiculação do conhecimento científico devido à sua rápida disseminação e fácil acesso ao texto completo.

O número expressivo de publicações em anais de congressos demonstra a relevância e o interesse de profissionais e acadêmicos pela temática Atividade Física e Envelhecimento. No entanto, a maior participação de pesquisadores da região Sudeste induz a interpretação de que maior quantidade de pesquisas e/ou número de eventos sejam também realizadas na região Sudeste.

Quanto ao número de artigos aceitos para publicação, a região Sudeste também possui maior número, quando comparado com as outras regiões. Observa-se que a região Nordeste assume o segundo lugar neste quesito quando a comparação é feita de forma absoluta. Por outro lado, em caso de comparação relativa ao número de GPAFEs, o número de artigos aceitos para publicação por pesquisadores da região Nordeste é o dobro em relação ao Sudeste e nove vezes maior em relação à região Sul. Embora haja limitação temporal para registro da produção científica, atualização da plataforma Lattes por parte dos pesquisadores e dificuldade em estabelecer a vinculação da produção à área da Atividade Física e Envelhecimento, estes dados refletem o crescimento da produção científica na região Nordeste do país.

Quando a publicação científica dos líderes foi analisada por subáreas de conhecimento, observa-se que a da Educação Física apresenta maior produção. A Organização Mundial da Saúde enfatiza a prática regular de atividade física como estratégia para promoção da saúde e envelhecimento saudável (WHO, 2004; 2005), dessa forma, espera-se maior envolvimento da subárea Educação Física na produção e veiculação do conhecimento nesta temática. 
Os estudantes formam a maioria dos integrantes dos GPAFEs. A inserção do aluno, principalmente o graduando, pode ser um fator estimulante para formação de novos pesquisadores de forma a atuar na produção de conhecimento. De acordo com Teixeira (2005), a expansão da produção de pesquisas é resultado de ações que visaram a formação e aperfeiçoamento de pesquisadores.

\section{Conclusão}

O presente estudo mostrou que a discussão da temática Atividade Física e Envelhecimento ainda não está disseminada no país. Observou-se uma evolução gradativa na criação dos GPAFEs em um período de cinco anos. A publicação de artigos em periódicos científicos pelos GPAFEs tem sido o principal meio para veiculação do conhecimento produzido e isso foi verificado para todas as subáreas do conhecimento de abrangência dos grupos analisados.

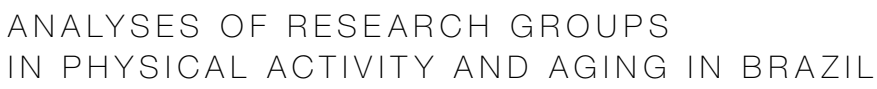

Regular physical activity has been recommended by experts in the field of aging because of its health benefits. The aim of this study was to analyze and characterize the research groups in physical activity and aging in Brazil, according to geographical distribution. A systematic search was conducted in the directory of research groups of the National Council for Scientific and Technological Development about the issue: "physical activity and aging". The research groups in physical activity and aging in Brazil certificates are distributed in three regions of Brazil: Southeast $(n=8)$, South $(n=6)$ and Northeast $(n=4)$. Articles published in journals represent 69.7\% $(n=639)$ of the scientific production of the Southeast region, $65.0 \%(n=39)$ of the South region and $64.9 \%(n=126)$ of the Northeast region. There was a gradual increase in the number of research groups in physical activity and aging in Brazil, but these groups are not distributed throughout the country.

keywords

Physical activity. Aging. Research. Scientific. Health Sciences. 
ALMEIDA, Osvaldo P. et al. 150 minutes of vigorous physical activity per week predicts survival and successful ageing: a population-based 11-year longitudinal study of 12 201 older Australian men. British Journal of Sports Medicine, London, v. 48, n. 3, p. 220-225, Feb. 2014.

ANDRÉA, Fernando et al. A atividade física e o enfrentamento do estresse em idosos. Einstein, São Paulo, v. 8, n. 4, p. 419-22, out./dez. 2010.

BALBÉ, Giovane Pereira et al. Produção científica sobre atividade física e envelhecimento em programas brasileiros de pós-graduação em Educação Física. Movimento, Porto Alegre, v. 18, n. 1, p. 261-267, jan./mar. 2012.

BRASIL. Ministério da Ciência e Tecnologia. Perguntas frequentes - Diretório dos Grupos de Pesquisa no Brasil, 2013a. Disponível em: <http://dgp.cnpq.br/censos/perguntas/ perguntas.htm\#5>. Acesso em: 05 abr. 2013.

Ministério da Educação - Coordenação de Aperfeiçoamento de Pessoal de Nível Superior. Relação de Cursos Recomendados e Reconhecidos. Grande área Ciências da Saúde, área Educação Física, 2013b. Disponível em: <http://www.capes. gov.br/cursos-recomendados>. Acesso em: 05 abr. 2013.

BROWN, Wendy et al. Physical activity and all-cause mortality in older women and men. British Association of Sport and Medicine, Loughborough, v. 46, n. 9, p. 664-668, Jul 2012.

GILLUM, Richard F.; OBISESAN, Thomas O. Physical activity, cognitive function, and mortality in a US national cohort. American College of Epidemiology, New York, v. 20, n. 4, p. 251-257, Apr. 2010.

INSTITUTO BRASILEIRO DE GEOGRAFIA E ESTATÍSTICA. Censo Demográfico 2010. IBGE: 2010a. Disponível em: <http://www.censo2010.ibge.gov.br/sinopse/webservice/>. Acesso em: 12 mar. 2013.

Projeção da População do Brasil por sexo e idade: 2000-2060. IBGE: 2013. Disponível em: <http://www.ibge.gov.br/home/estatistica/populacao/projecao_da_populacao/2013/>. Acesso em: 12 nov. 2013.

Sintese de indicadores sociais: Uma análise das condições de vida da população brasileira 2010, IBGE: 2010b. Disponível em: <http://www.ibge.gov.br/home/ estatistica/populacao/condicaodevida/indicadoresminimos/sinteseindicsociais2010/ SIS_2010.pdf>. Acesso em: 25 fev. 2013.

KALACHE, Alexandre. O mundo envelhece: é imperativo criar um pacto de solidariedade social. Ciência \& Saúde Coletiva, Rio de Janeiro, v. 13, p. 1107-1111, jul./ago. 2008.

LEBRÃO, Maria Lúcia. O envelhecimento no Brasil: aspectos da transição demográfica e epidemiológica. Saúde Coletiva, São Paulo, v. 4, n. 17, p. 135-140, set. 2007.

MATSUDO, Sandra Mahecha. Atividade física na promoção da saúde e qualidade de vida no envelhecimento. Revista Brasileira de Educação Física e Esporte, São Paulo, v. 20, Suplemento n.5, p. 135-137, set. 2006.

MATSUDO, Sandra Mahecha; MATSUDO, Victor Keihan Rodrigues; BARROS NETO, Turíbio Leite. Atividade física e envelhecimento: aspectos epidemiológicos. Revista Brasileira de Medicina do Esporte, Niterói, v. 7, n. 1, p. 2-13, jan./fev. 2001.

MENDES, Edmar Lacerda; ANDAKI, Alynne Christian Ribeiro; ANDAKI JÚNIOR, Roberto. Grupos de Pesquisa em atividade Física e Saúde no País: quem são e em que lugares estão localizados. In: VIRTUOSO JÚNIOR, Jair Sindra. Interfaces multidisciplinares na saúde aplicadas na formação do profissional em Educação Física. Uberaba: UFTM, 2011. p. 29-37. 
PRADO, Shirley Donizete; SAYD, Jane Dutra. A pesquisa sobre envelhecimento humano no Brasil: grupos e linhas de pesquisa. Ciência \& Saúde Coletiva, Rio de Janeiro, v. 9, n. 1, p. 57-67, 2004.

SCULLY, Tony. Demography: to the limit. Nature, London, v. 492, n. 7427, p. S2-S3, Dec. 2012

TEIXEIRA, Maria da Glória. IV Plano Diretor para o Desenvolvimento da Epidemiologia no Brasil. Revista Brasileira de Epidemiologia, São Paulo, v. 8, n. 3, p. 231-233, set. 2005.

VERAS, Renato. Envelhecimento populacional contemporâneo: demandas, desafios e inovações. Revista de Saúde Pública, São Paulo, v. 43, n. 3, p. 548-54, maio/jun. 2009.

WHO. Word Health Organization. Global strategy on diet and physical activity. Geneva: 2004. Disponível em: <http://www.who.int/dietphysicalactivity/strategy/eb11344/strategy_english_web.pdf>. Acesso em: 19 nov. 2012.

Envelhecimento ativo: uma política de saúde. Brasília: 2005. Disponível em: <http://www.prosaude.org/publicacoes/diversos/envelhecimento_ativo.pdf>. Acesso em: 15 set. 2012.

Recebido em: 31/08/2013

Aceite Final: 30/10/2014 